\title{
Comparison of the Changes in the Range of Motion on the Knee Joint According to the Contraction Intensity During Evjenth-Hamberg Stretching in Healthy Subjects: A Cross-Sectional Pilot Study
}

\author{
Do-Kwan Kim, PT, MSc • Chang-Yong Kim, PT, PhD ${ }^{1}$ Dong-Kwon Seo, PT, PhD ${ }^{2} \cdot$ \\ Byoung-Kwon Lee PT, $\mathrm{PhD}^{2 \dagger}$ \\ SEOL Orthopedics Surgery \\ ${ }^{1}$ Scientific Instruments Reliability Assessment Center, Korea Basic Science Institute \\ ${ }^{2}$ Deptartment of Physical Therapy, Konyang University
}

Received: March 29, 2019 / Revised: April 2, 2019 / Accepted: May 30, 2019

(c) 2019 J Korean Soc Phys Med

\section{| Abstract |}

PURPOSE : This study compared the changes in the range of motion (ROM ) on the knee joint according to three different isometric contraction intensities when applying Evjenth-Hamberg stretching (EHS) to the hamstring muscles of healthy subjects.

METHODS: Forty eight healthy subjects aged between 20 and 39 years were allocated randomly to four groups; performing EHS at $10 \%$ maximum voluntary isometric contraction (MVIC) group, EHS at 50\% MVIC group, EHS at $100 \%$ MVIC group, and control group (CG) pre-, and post-intervention. The flexibility of the hamstring muscles was evaluated using a digital goniometer for measuring the knee joint ROM motion. A Chattanooga stabilizer was used

$†$ Corresponding Author : Byoung-Kwon Lee miraclept1234@daum.net, http://orcid.org/0000-0002-7251-6108 This is an Open Access article distributed under the terms of the Creative Commons Attribution Non-Commercial License (http://creativecommons.org/licenses/by-nc/3.0) which permits unrestricted non-commercial use, distribution, and reproduction in any medium, provided the original work is properly cited. to adjust the MVIC intensity by $10 \%, 50 \%$, and $100 \%$, respectively.

RESULTS: These results show that the pre- and postintervention knee joint ROM were significantly different in all four groups $(\mathrm{p}<.05)$. The post-intervention knee joint ROM showed a significant difference between the $100 \%$ MVIC group and non-MVIC group $(\mathrm{p}<.05)$. The knee joint ROM difference between the pre- and post-interventions was similar in the $10 \%$ MVIC and 50\% MVIC groups but significant differences were observed among the other groups $(\mathrm{p}<.05)$.

CONCLUSION: EHS of the hamstring muscles was effective in improving the knee joint ROM with an isometric contraction intensity of $10 \%, 50 \%$, or $100 \%$ MVIC, and of these, $100 \%$ MVIC was most effective.

Key Words: Evjenth-Hamberg stretching, Hamstring muscle, Isometric contraction intensity, Knee joint, Range of motion 


\section{Introduction}

In modern-day individuals, changes in living environments due to industrialization, decreases in physical activity and mobility, and aging are causing problems with muscle extensibility by depleting the muscle fibers and elastic components in the muscle [1]. In particular, owing to the popularization of computers, seated workers who are regular computer users increasingly complain of musculoskeletal system pain in the cervical spine and shoulders [2], as well as hamstring muscle shortening, which reduce the flexibility, leading to impairments in the activities of daily living (ADL) [3,4]. Flexibility is an important physical factor associated with health. The modern environment, which necessitates long periods of sitting and involves a lack of regular physical activity, is a major factor that causes a decrease in flexibility [5].

Flexibility can be divided broadly into active and passive flexibility. Active flexibility is affected by the tissue resistance and the extent of joint movement as a result of muscle contraction. Passive flexibility is affected directly by the extensibility of the muscles and connective tissue crossing the joint. Dynamic flexibility, which is a type of active flexibility, can be described in terms of the ability to move a body part rapidly. Static flexibility, which is a type of passive flexibility, can be described in terms of the range of motion (ROM) of a body part [6]. A loss of muscle flexibility causes changes in the length and tension of the muscle [7], which can in turn lead to reduced muscular strength. Hence, proper stretching can help improve the muscular strength [8]. Good flexibility improves the quality of exercise by increasing the ROM and massively reduces the risk of injury during exercise or ADL [9].

Stretching is used in sports medicine to prevent injury, reduce pain and muscle tension after physical activity, and increase the ROM. In rehabilitation, stretching is used not only to improve the ROM, but also restore the function after acute injuries for pain control and tissue immobilization [10]. Stretching is often used before exercise, as a warm-up of overly tense muscles [11], or to improve the muscle and joint flexibility [12]. To restore the limited ROM due to shortening, scar tissue formation, or hypo-mobility structure, stretching can be used with varying intensity, duration, and frequency, based on the clinical judgment of the physical therapist $[8,13]$. The advantages of stretching are that it not only improves flexibility, but also relieves excessive muscle tension, alleviates muscle pain, enhances blood circulation, and reduces the muscle resistance by preventing excessive adhesion between the muscles [14].

Stretching is effective in increasing the maximum torque of the hamstring muscles [15]. Flexibility and muscular strength improve when athletes perform hold-relax stretching [16]. Static stretching alone of the quadriceps femoris muscle was reported to improve the isometric muscular strength by $10 \%$ [17], and stretching and muscular strength have been shown to be associated [13]. Stretching after isometric contraction has been reported to not only improve flexibility, but also alter the muscle characteristics, such as increasing muscle activity [16]. Therefore, these studies show that a combination of stretching and isometric contraction is effective in improving the muscular strength. Kim [18] examined the effects of the hold-relax method on pain and flexibility when performed voluntarily in call-center workers, whereas Davis et al. [19] compared the effects of auto stretching, static stretching, dynamic stretching, and proprioceptive neuromuscular facilitation (PNF) stretching on the hamstring muscle flexibility. Nagarwal et al. [20] compared the effects of the hold-relax and contract-relax-antagonist muscle contraction methods. Oh [21] compared the effects of direct and indirect methods for hold-relax stretching.

Of these, the Evjenth-Hamberg stretching (EHS) method combines static stretching with isometric contraction of the agonist and antagonist muscles [22]. The concept that 
stimulation of both muscles is important for restoring the normal function in the antagonist muscle was introduced using the EHS method [23]. In relation to this method, Park [24] applied EHS to the sternocleidomastoid, upper trapezius, and pectoralis major muscles and found that it was effective in improving the forward head posture; the isometric contraction intensity for EHS was not reported. When this experiment was designed, previous studies were insufficient in determining which intensities to compare. Therefore, 100\% (maximum) [22,23,25], 50\% (median), and $10 \%$ (minimum) of the maximum voluntary isometric contraction (MVIC) were selected arbitrarily.

Most studies compared the effects between the PNF stretching methods or between static stretching, dynamic stretching, PNF stretching, and EHS. On the other hand, few studies have compared the effects of the agonist muscle isometric contraction intensity on the outcomes of EHS, which combines static stretching with isometric contraction of the agonist and antagonist muscles. Therefore, in the present study, EHS was applied with agonist muscle isometric contraction intensities of $10 \%, 50 \%$, and $100 \%$ MVIC, and the effects on active knee extension ROM were compared. The aim was to determine the most effective contraction intensity for physical therapists utilizing EHS in clinical practice.

\section{Methods}

\section{Participants}

Based on the inclusion and exclusion criteria for this study, 48 healthy adult men, aged 20-39 years who were living in Daegu, South Korea, were enrolled in this study. The study was approved by the Human Research Sciences of Local Ethics Committee and registered with the University Clinical Trials Registry. Prior to the experiment, the subjects filled out a consent form, provided information regarding their general characteristics, and underwent an MVIC test. All subjects were assigned randomly to the
EHS at $10 \%$ MVIC group, EHS at 50\% MVIC group, EHS at $100 \%$ MVIC group, or control group. The right hamstring muscle group was selected as the intervention muscle to measure the equality of participants in this study [26]. The inclusion criteria were as follows: 1) individuals who thoroughly understood the study content, aims, procedure, and subject rights protection, and gave their voluntary consent to participate; 2) individuals with no disease (pain) in the lumbar spine, right hip joint, or knee joint [26]; 3) individuals with a normal passive ROM of the right knee joint $[25,26]$; 4) individuals with no sciatic nerve signs in active knee extension [26,27]; 5) individuals who did not normally perform at least 30 minutes of hamstring muscle stretching or strength training per day [26]. The exclusion criteria were as follows: 1) individuals could extend the knee joint fully in active knee extension $[26,27]$ and 2) individuals with no current disease but had a history of surgery [25].

\section{Outcome Measures}

The methods of measuring stretching included a visual inspection, ROM, a goniometer, or a flexible ruler, but the most commonly used method was to measure the ROM with a goniometer [27,28]. The axis of the goniometer was aligned with the lateral epicondyle of the femur, the proximal arm points along the femur toward the greater trochanter of the femur, and the distal arm points along the lower leg toward the lateral malleolus [29]. When measuring the knee joint ROM, before measuring the angle, a 30-cm stick was fixed to one arm of the goniometer (two arms) with tape to make a total length of $50 \mathrm{~cm}$. The stick was attached to reduce the distance between the goniometer and the body markers (greater trochanter of the femur and lateral malleolus of the fibula), making the measurement easier and more accurate [27]. The goniometer used in the present experiment was the $\mathrm{A}$ Baseline Digital Absolute + Axis Goniometer, which is a digital goniometer manufactured by Preston (USA), with 


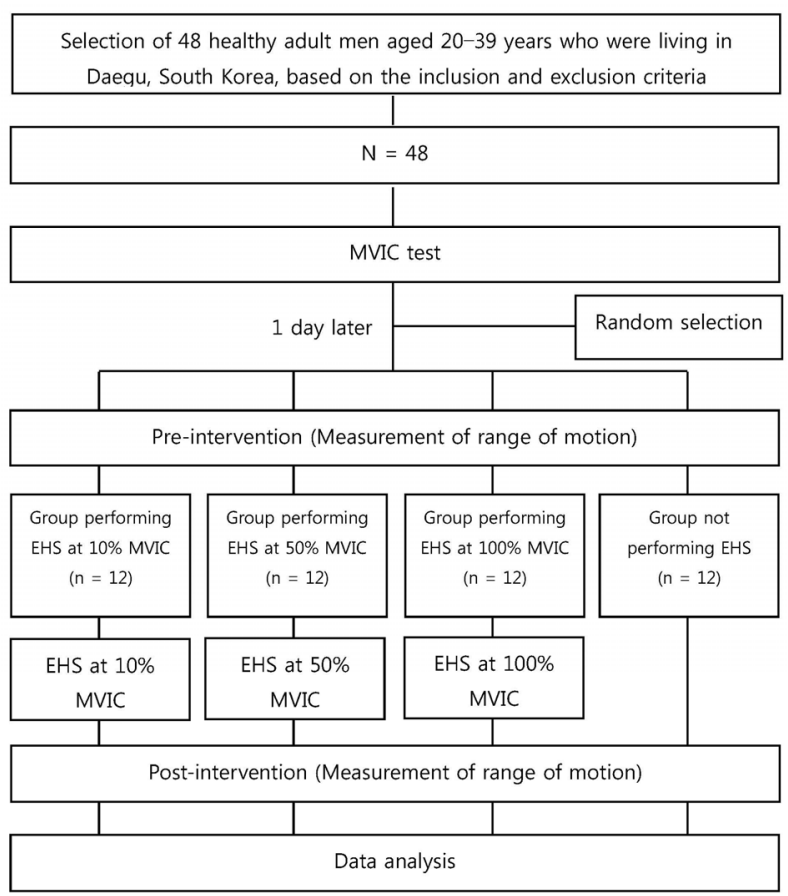

Fig. 1. Experimental procedures in the present study (EHS: Evjenth-Hamberg stretching, MVIC: maximum voluntary isometric contraction)

a reliability of $r=.47-.69$ [30].

The subject was laid on the table in the supine position, and both the ASIS and the left femoral region were fixed to the table with belts. The subject was instructed to actively flex the right hip joint to $90^{\circ}$ (perpendicular to the table), and the examiner then held the femur in place. A T-shape was used to maintain a constant hip flexion angle of $90^{\circ}$. The horizontal arm of the " $\mathrm{T}$ " was placed along the table, and the vertical arm was positioned to pass through the greater trochanter and lateral epicondyle of the femur. The subject was then instructed to perform active knee extension. Here, the ankle joint was dorsiflexed to avoid interfering with knee joint extension. The end range of extension was verified where the subject experienced muscle clonus, and the extension angle was measured at this point. This method has been used recently in many studies [27,31].

\section{Experimental processing}

Fig. 1 outlines the test procedures. In EHS, isometric contraction intensities of $10 \%, 50 \%$, and $100 \%$ MVIC were used, and the effects on the knee joint ROM were compared. The MVIC measuring method reported by Kwak [26] was adapted to suit the hamstring muscles. MVIC was measured using a Chattanooga stabilizer (Chattanooga Group Inc., Hixson, USA) before allocating the subjects randomly to one of four groups (10\% MVIC group, 50\% MVIC group, $100 \%$ MVIC group, and control group). The right knee joint ROM was then measured as the pre-test in all the subjects. After this, the subjects performed EHS (Fig. 2) at an intensity of $10 \%$ MVIC in the $10 \%$ MVIC group, $50 \%$ MVIC in the $50 \%$ MVIC group, and $100 \%$ MVIC in the 100\% MVIC group [25]. The right knee joint ROM was measured immediately afterward as the post-intervention. The subject's right heel and leg were placed on the examiner's left shoulder. A Chattanooga stabilizer was 


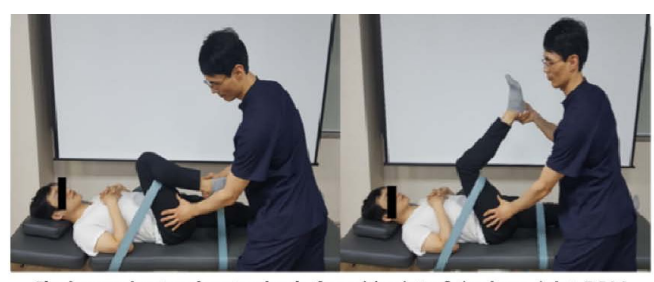

Flexion and extension to check the midpoint of the knee joint ROM

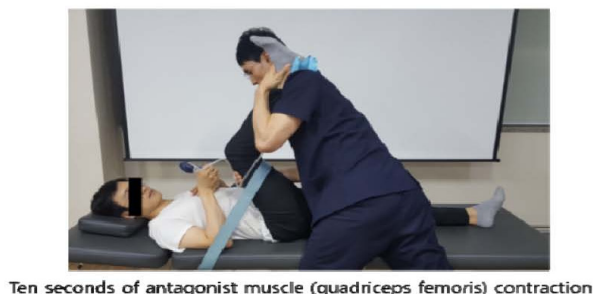

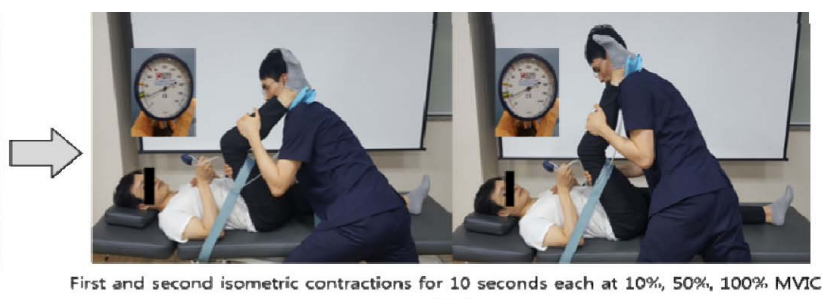

First and second isometric contractions for 10 seconds each at 10\%, 50\%, 100\% MVC
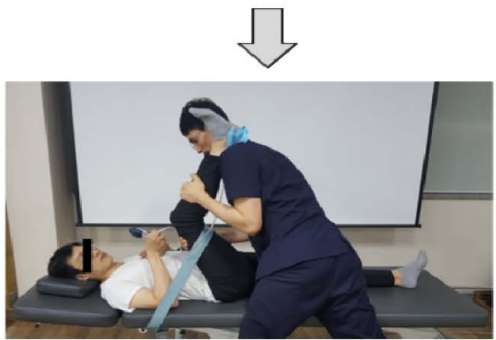

Static stretching for 30 seconds

Fig. 2. Described procedures for three different isometric contraction intensities when applying Evjenth-Hamberg stretching to the hamstring muscles

folded twice and placed between the subject's heel and the examiner's left shoulder. The subject held the dial of the Chattanooga stabilizer so that he/she could view the pressure directly. The examiner held the anterior surface of the subject's right leg with the left hand immediately distal to the knee joint and used the right hand to hold the anterior surface of the leg immediately proximal to the knee joint for stability. The subjects in the control group did not perform EHS, and the right knee joint ROM was measured immediately as a post-intervention. The measurements were performed by a licensed physical therapist with 12 years clinical experience and an orthopedic manual therapist (OMT) certificate for the OMT Kaltenborn-Evjenth concept. Training sessions for all groups were carried out at the university laboratory. All participants received approximately an hour training session and the mean of three attempts was calculated.

\section{Statistical analysis}

Statistical analysis of the data was performed using IBM SPSS 22.0 software (IBM SPSS Inc., Chicago, IL, USA). The values in each group are expressed as the mean \pm standard deviation. Parametric methods were used because the samples involved in the present study were represented by normal distribution curves in the Shapiro-Wilk test. The subjects' general characteristics were analyzed using the one-way analysis of variance (ANOVA). A paired samples t-test was used to compare the pretest and posttest differences within each group, and one-way ANOVA was used to examine the differences between groups after the intervention. The Bonferroni's post-hoc test was used to identify the differences among the groups for each intervention time. To determine the statistical significance of the data collected from the subjects, the significance level was set to $\mathrm{p}<.05$.

\section{Results}

\section{General characteristics of the subjects}

The study subjects consisted of 48 healthy adult men aged 20-39 years. The subjects were divided randomly into a $10 \%$ MVIC group $\left(\mathrm{n}_{1}=12\right), 50 \%$ MVIC group $\left(\mathrm{n}_{2}=12\right)$, $100 \%$ MVIC group $\left(\mathrm{n}_{3}=12\right)$, and Non MVIC (NMVIC) group $\left(\mathrm{n}_{4}=12\right)$. Table 1 lists the subjects' general charac 
Table 1. General Characteristics of the Subjects

\begin{tabular}{cccccc}
\hline & $\mathrm{A}\left(\mathrm{n}_{1}=12\right)$ & $\mathrm{B}\left(\mathrm{n}_{2}=12\right)$ & $\mathrm{C}\left(\mathrm{n}_{3}=12\right)$ & $\mathrm{D}\left(\mathrm{n}_{4}=12\right)$ & $\mathrm{p}$-Value \\
\hline Age $(\mathrm{y})$ & $30.581 \pm 5.362$ & $30.665 \pm 4.456$ & $30.661 \pm 4.755$ & $30.662 \pm 4.942$ & 1.000 \\
Height $(\mathrm{cm})$ & $177.581 \pm 5.313$ & $175.006 \pm 4.697$ & $172.833 \pm 3.951$ & $176.912 \pm 5.851$ & .101 \\
Weight $(\mathrm{kg})$ & $76.081 \pm 7.624$ & $71.581 \pm 8.684$ & $72.668 \pm 9.993$ & $74.912 \pm 4.713$ & .503 \\
\hline
\end{tabular}

Values are expressed as the mean \pm standard deviation.

A: MVIC $10 \%$, B: MVIC 50\%, C: MVIC 100\%, D: NMVIC.

MVIC: maximum voluntary isometric contraction.

NMVIC: non-maximum voluntary isometric contraction.

$* \mathrm{p}<.05$.

Table 2. Comparison of the Pretest, Posttest, and Difference Values Between the Groups

(unit: ${ }^{\circ}$ )

\begin{tabular}{cccc}
\hline Group & Pre-intervention & Post-intervention & Difference value \\
\hline A & $136.715 \pm 9.013$ & $144.251 \pm 10.105$ & $7.504 \pm 2.572$ \\
B & $138.584 \pm 4.711$ & $146.373 \pm 4.774$ & $7.751 \pm .625$ \\
C & $138.252 \pm 9.094$ & $151.753 \pm 6.912$ & $13.558 \pm 2.814$ \\
D & $140.581 \pm 4.839$ & $141.331 \pm 4.846$ & $.752 \pm .755$ \\
p-Value & .631 & $.006^{*}$ & $.000^{\dagger}$ \\
\hline
\end{tabular}

Values are expressed as the mean \pm standard deviation.

A: MVIC 10\%, B: MVIC 50\%, C: MVIC 100\%, D: NMVIC.

MVIC: maximum voluntary isometric contraction.

NMVIC: non-maximum voluntary isometric contraction.

*: Significant difference in post-intervention values between the groups $(\mathrm{p}<.05)$.

$t$ : Significant difference in difference values between the groups $(p<.05)$.

teristics. The age (years), height (centimeters), and weight (kilograms) of the subjects in each group were examined. A test of the homogeneity among the groups for these three variables revealed no significant differences ( $p>05)$.

\section{Comparison of the pre- and post-intervention knee joint ROM among the groups}

Table 2 lists the pre- and post-intervention knee joint ROM of the subjects. The pre-intervention knee joint ROM did not show any significant differences among the four groups ( $>$.05). On the other hand, the post-intervention knee joint ROM showed significant differences among the groups $(\mathrm{p}<.05)$. In post hoc testing using the Bonferroni method, a significant difference was only detected between the $100 \%$ MVIC group and NMVIC group ( $p<.05$ ) (Fig. 3).
The pre- and post-intervention knee joint ROM difference values also showed significant differences among the groups $(\mathrm{p}<.05)$. In post hoc testing using the Bonferroni method, no statistically significant difference was found between the $10 \%$ and $50 \%$ MVIC groups ( $>.05$ ), but significant differences were detected among the other groups ( $<$.05) (Fig. 4).

\section{Comparison of the pre- and post-intervention knee joint ROM within the groups}

All four groups showed significant within-group differences between pre-intervention and post-intervention knee joint ROM $(\mathrm{p}<.05)$ (Table 3). 


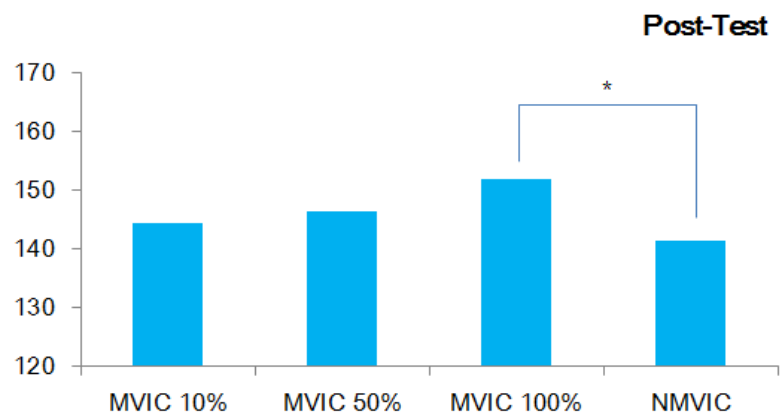

Fig. 3. Post hoc test results for the differences in posttest values between the groups (MVIC: maximum voluntary isometric contraction, NMVIC: non-maximum voluntary isometric contraction, ${ }^{*} \mathrm{p}<$.05.)

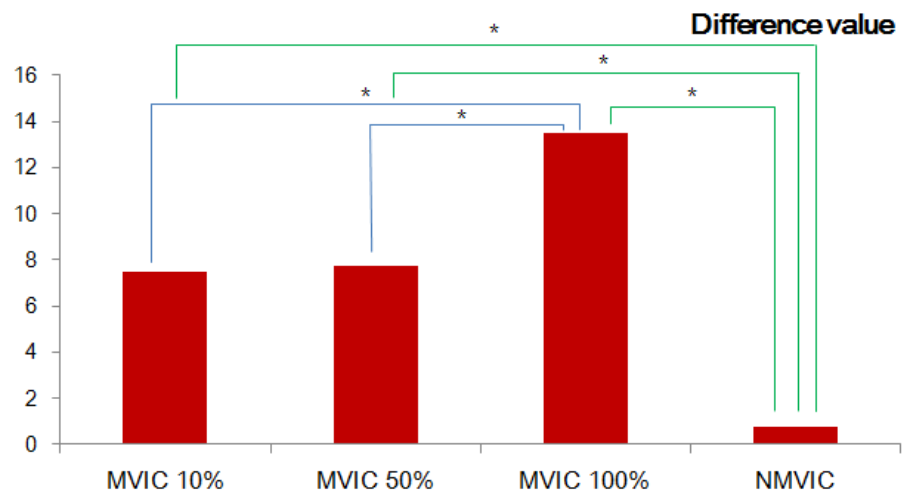

Fig. 4. Post hoc test results for the differences in difference values between the groups (MVIC: maximum voluntary isometric contraction, NMVIC: non-maximum voluntary isometric contraction, ${ }^{*} \mathrm{p}<.05$.)

Table 3. Comparison of Pretest and Posttest Values within the Groups

(unit: ${ }^{\circ}$ )

\begin{tabular}{ccccc}
\hline Group & Pretest & Posttest & p-Value & $t$ \\
\hline A & $136.751 \pm 9.013$ & $144.251 \pm 10.101$ & $.000^{*}$ & -10.085 \\
B & $138.581 \pm 4.715$ & $146.333 \pm 4.775$ & $.000^{*}$ & -43.191 \\
C & $138.252 \pm 9.098$ & $151.751 \pm 6.919$ & $.000^{*}$ & -16.629 \\
D & $140.583 \pm 4.838$ & $141.335 \pm 4.841$ & $.003^{*}$ & -3.447 \\
\hline
\end{tabular}

Values are expressed as the mean \pm standard deviation.

A: MVIC 10\%, B: MVIC 50\%, C: MVIC 100\%, D: NMVIC.

MVIC: maximum voluntary isometric contraction.

NMVIC: non-maximum voluntary isometric contraction.

$* \mathrm{p}<.05$.

\section{Discussion}

The isometric contraction intensities of $10 \%, 50 \%$, and
$100 \%$ MVIC were used, and the effects of the different isometric contraction intensities on the knee joint ROM were compared. Significant differences in the post-EHS 
knee joint ROM were detected between the groups, and post hoc testing using the Bonferroni method revealed a significant difference only between the $100 \%$ MVIC group and NMVIC group $(p<05)$. Statistically significant differences in the pre- and post-EHS knee joint ROM difference values were also observed between the groups $(p<.05)$. Therefore, when performing EHS, an isometric contraction intensity of $100 \%$ MVIC was more effective in restoring the hamstring muscle flexibility than $10 \%$ or $50 \%$ MVIC, and no significant differences in the effects of $10 \%$ and $50 \%$ MVIC were found. In addition, the $10 \%$, $50 \%$, and $100 \%$ MVIC groups all showed a significant increase in the knee joint ROM following EHS. This shows that EHS is effective in restoring the hamstring muscle flexibility, irrespective of the isometric contraction intensity.

Marek et al. [32] implemented static and PNF stretching of the knee extensors in 19 healthy, active college students. When they compared the peak torque, mean power output, active ROM, and passive ROM, the authors observed decreases in peak torque and mean power output, and improvements in the active and passive ROM for both static and PNF stretching. Weng et al. [33] divided 132 subjects with osteoarthritis (Altman grade 2) in both knee joints into three groups and implemented isokinetic muscular strengthening exercise, isokinetic muscular strengthening exercise with static stretching, and isokinetic muscular strengthening exercise with PNF stretching. Using a visual analog scale, Lequesne' $s$ index, knee joint ROM, and peak muscle torque, they reported a massive decrease in pain and disability, an increase in knee joint ROM, and improvements in muscular strength for both static and PNF stretching, with PNF stretching being more effective in all areas. Li et al. [34] divided 39 healthy subjects into two groups and compared straight leg raising and active knee extension angle between a group with and a group without participation in stretching of the hamstring muscles. The stretching group showed improvements of $14.28^{\circ}$ in straight leg raising and $7.95^{\circ}$ in active knee extension. Swank et al. [35] reported that stretching after isometric contraction can not only improve the ROM, but also alter the transverse cross-sectional area of the muscle fibers and muscle characteristics. Powers and Howley [36] reported that an elevated intramuscular temperature due to isometric contraction and additional isometric contraction of the agnostic muscle in static stretching could improve the flexibility by increasing the synthesis of proteins that prevent muscle atrophy. In addition, the intramuscular temperature induced by an isometric contraction promotes the relaxation of antagonist muscles, such as quadriceps muscles, and increases the range of normal joint motion. Knight et al. [37] reported that increasing the tissue temperature to $45^{\circ} \mathrm{C}$ before stretching increases the tissue length while minimizing the risk of injury due to physiological advantages, such as increased blood flow, increased elasticity of collagen tissue, elevated pain threshold, and alleviation of muscle stiffness. When a muscle becomes warm, it stretches more readily; rather than deliberately applying exogenous heat, it is better physiologically to perform warm-up exercises. For this reason, all treatment begins with warm-up exercises. The most effective and specific warm-up exercises induce contraction while applying resistance. Stronger contractions increase the effects of warm-up exercises [25]. This provides indirect evidence of the efficiency of adding antagonist muscle contraction to stretching [38]. Therefore, the 10-second agonist and antagonist muscle isometric contractions used in EHS are believed to help improve muscle flexibility by increasing the intramuscular temperature [23].

EHS, in which antagonist muscle contraction is added to stretching, is often used clinically as a therapeutic approach to increase the ROM, and many studies have demonstrated its effectiveness. EHS has been shown to be more effective in increasing the ROM than static stretching, possibly because the relaxation effect after isometric contraction is larger in EHS than in static 
stretching. Jung [39] implemented isometric contraction of the agnostic muscle and the antagonist muscle alongside static stretching of the hamstring muscle, and reported increases in both the ROM and muscular strength. Kim [40] implemented static and PNF stretching, which adds an isometric contraction, for the hamstring muscles and examined the duration for which flexibility had been maintained. In the static stretching group, flexibility decreased after 15 minutes. In the PNF stretching group, however, flexibility increased and remained higher even after 30 minutes. Björklund et al. [41] suggested that isometric contraction before stretching, as an important factor in improving flexibility, might promote the sensory adaptation.

On the other hand, each study using EHS focused only on an isometric contraction of the agonist or antagonist muscle, and most studies only examined the effects of EHS, in which an isometric contraction was added. Several studies did not even mention the contraction intensity. Therefore, this study compared the difference in the ROM when EHS was performed at different isometric contraction intensities to determine the more efficient contraction intensity when using EHS clinically. When the study was designed, previous studies to decide which contraction intensities to compare were lacking, so values of $100 \%$ (maximum) [22,23,25], 50\% (median), and 10\% (minimum) MVIC were selected arbitrarily. In studies that examined the effects of stretching, several factors influenced the results, including the study duration, number of subjects, and restricted muscle length differences between subjects [20]. Even more variables could be involved when EHS is used in actual clinical practice. In addition to the isometric contraction intensity, differences could arise according to the contraction time, post-contraction relaxation time, contraction frequency, contraction intensity during stretching, and the experience and capability of the physical therapist. For this to become a more efficient stretching method in the future, additional studies will be needed to explore these variables.

This study had several limitations. First, as a crosssectional pilot study, only the immediate effect was measured and only a temporary increase for healthy adult men was reported (Smith 1994). Second, the reliability of the digital goniometer used to measure the ROM in this study was too low $(\mathrm{r}=.47-.69)$, and the method of measurement was unscientific because the results can vary according to the examiner. Third, evidence for a comparison between 10\% MVIC, 50\% MVIC, and 100\% MVIC is rare because previous studies that compared the effects of EHS at different isometric contraction intensities are rare. That is, different results can be obtained depending on the degree of hamstring shortening. Therefore, it is necessary to conduct a comparative study on the MVIC intensity, together with scientific test and evaluation criteria to clearly select the subjects with hamstring shortening. Finally, during static stretching in EHS, objectivity for the stretching end point (soft end feel) is lacking. Future research will compare the treatment effects when other variables are altered, examine the duration for which the treatment effect is maintained, and account thoroughly for the limitations of the present study.

\section{Conclusion}

This study showed that when using EHS on shortened hamstring muscles, isometric contraction intensities of $10 \%, 50 \%$, and $100 \%$ MVIC are all effective in restoring hamstring muscle flexibility. In terms of the differences in effectiveness between the different isometric contraction intensities, 100\% MVIC was more effective in restoring the hamstring muscle flexibility than $10 \%$ or $50 \%$ MVIC. The use of EHS with 100\% MVIC will be more effective clinically, but further research on the clinical application and long-term effects on more muscle groups will be needed. 


\section{References}

[1] Faulkner JA, Larkin LM, Claflin DR, et al. Age-related changes in the structure and function of skeletal muscles. Clin Exp Pharmacol Physiol. 2007;34(11):1091-6.

[2] Mekhora K, Liston CB, Nanthavanij S, et al. The effect of ergonomic intervention on discomfort incomputer users with tension neck syndrome. Int Journal of IndusErg. 2000;26(2000):367-79.

[3] Ong CN, Chia SE, Jeyaratnam J, et al. Musculoskeletal disorders among operators of visual display terminals. Scand J Work Environ Health. 1995;21(1):60-4.

[4] Kim, SY. Comparison of six tests for assessing hamstring muscle length. J Korean Acad Orthop Man PhysiTher. 1999;5(1):39-51.

[5] Kendall FP, McCreary EK, Provance PG. Muscle Testing and Function (4 $4^{\text {th }}$ ed). Philadelphia. Lippincott, Williams and Wilkins. 1993.

[6] O'Sullivan SB, Schmitz TJ. Physical rehabilitation (5 ed). Philadelphia. FA Davis. 2006.

[7] Jo HS, Lee BM. Literature Review on the Effects of PNF, PIC, and MET Extension Techniques. J Sport Leisure Studies. 2005;23(5):379-90.

[8] Colby LA, Kisner C. Therapeutic exercise: Foundations and techniques. Philadelphia. FA Davis. 2007.

[9] Ogura Y, Miyahara Y, Naito H, et al. Duration of static stretching influences muscle force production in hamstring muscles. J Strength Cond Res. 2007;21(3):788-92.

[10] Halbertsma JP, Göeken LN. Stretching exercises: effect on passive extensibility and stiffness in short hamstrings of healthy subjects. Arch Phys Med Rehabil. 1994; 75(9):976-81.

[11] Dadebo B, White J, George KP. A survey of flexibility training protocols and hamstring strains in professional football clubs in England. Br J Sports Med. 2004;38(4): 388-94.

[12] Witvrouw E, Mahieu N, Danneels L, et al. Stretching and injury prevention: an obscure relationship. Sports
Med. 2004;34(7):443-9.

[13] Rubini EC, Costa AL, Gomes PS. The effects of stretching on strength performance. Sports Med. 2007;37(3):213-24.

[14] Baek HJ. Effects of Stretching Techniques on the Flexibility of Soleus Muscle. Master's Degree. Ewha womans University. 1997.

[15] Worrell TW, Smith TL, Winegardner J. Effect of hamstring stretching on hamstring muscle performance. J Orthop Sports Phys Ther. 1994;20(3):154-9.

[16] Handel M, Horstmann T, Dickhuth HH, et al. Effects of contract-relax stretching training on muscle performance in athletes. Eur J Appl PhysiolOccup Physiol. 1997;76(5):400-8.

[17] Hwang WH. Effects of quadriceps muscle flexion on functional activity in patients with degenerative knee osteoarthritis. Master's Degree. Sahmyook University. 2002.

[18] Kim YM. Effects of the use of the hold relax technique to treat female VDT workers with work-related neck-shoulder complaints. Korean J Occup Environ Med. 2009;21(1):18-27.

[19] Davis DS1, Ashby PE, MoCale KL, et al. The effectiveness of 3 stretching techniques on hamstring flexibility using consistent stretching parameters. J Strength Cond Res. 2005;19(1):27-32.

[20] Nagarwal AK, Zutshi K, Ram CS, et al. Improvement of hamstring flexibility: A comparison between two PNF stretching techniques. Int Journal of Sports Sci Eng. 2010;4(1):25-33.

[21] Oh YT. Effect of Hold-Relax Technique for College Students with Hamstring Shortening. J Korea Soc Physical Med. 2013;8(3):433-41.

[22] Lee HH. The Effects of Static Stretching and Evjenth-Hamberg Stretching on Range of Motion ok Knee Joint. Doctor's Degree. Yonsei University. 2003.

[23] Chon SC, Chang KY. The Effects of Evjenth-Hamberg Stretching on Active Range of Motion of the Hip Joint and the Pennation Angle. Korean Res Soc Physical Ther. 
2010;17(2):43-50.

[24] Park JH. The Effects of Evjenth-Hamberg Stretching and Static Stretching on Improvement of Forward Head Posture. Doctor's Degree. Yongin University. 2012.

[25] Evjenth O, Hamberg J. Muscle Stretching in Manual Therapy: The spinal column and the temporo-mandibular joint. Alfta. Sweden. Alfta Rehab. 1984.

[26] Kwak DH. Comparison of Changes in Range of Motion by the Intensity of Contraction When Applying Post Isometric Relaxation Technique. Doctor's Degree. Daegu Catholic University. 2014.

[27] Bandy WD, Irion JM, Briggler M. The effect of time and frequency of static stretching on flexibility of the hamstring muscles. Phys Ther. 1997;77(10):1090-6.

[28] Youdas JW, Krause DA, Egan KS, et al. The effect of static stretching of the calf muscle-tendon unit on active ankle dorsiflexion range of motion. JOrthop Sports Phys Ther. 2003;33(7):408-17.

[29] Funk DC, Swank AM, Mikla BM, et al. Impact of prior exercise on hamstring flexibility: a comparison of proprioceptive neuromuscular facilitation and static stretching. J Strength Cond Res. 2003;17(3):489-92.

[30] Carey MA, Laird DE, Murray KA, et al. Reliability, validity, and clinical usability of a digital goniometer. Work. 2010;36(1):55-66.

[31] Reurink G, Goudswaard GJ, Oomen HG, et al. Reliability of the active and passive knee extension test in acute hamstring injuries. Am J Sports Med. 2013;41(8):1757-61.

[32] Marek SM, Cramer JT, Fincher AL, et al. Acute Effects of Static and Proprioceptive Neuromuscular Facilitation Stretching on Muscle Strength and Power Output. J Athl Train. 2005;40(2):94-103.

[33] Weng MC, Lee CL, Chen CH, et al. Effects of different stretching techniques on the outcomes of isokinetic exercise in patients with knee osteoarthritis. Kaohsiung J Med Sci. 2009;25(6):306-15.

[34] Li Y, McClure PW, Pratt N. The effect of hamstring muscle stretching on standing posture and on lumbar and hip motions during forward bending. Phys Ther. 1996;76(8):836-45.

[35] Swank AM, Funk DC, Durham MP, et al. Adding weights to stretching exercise increases passive range of motion for healthy elderly. J Strength Cond Res. 2003;17(2): 374-8.

[36] Powers SK, Howley ET. Exercise physiology: Theory and application to fitness and performance. McGraw-Hill. 2004.

[37] Knight CA, Rutledge CR, Cox ME, et al. Effect of superficial heat, deep heat, and active exercise warm-up on the extensibility of the plantar flexors. Phys Ther. 2001;81(6):1206-14.

[38] Safran MR, Garrett WE Jr, Seaber AV, et al. The role of warm up in muscular injury prevention. Am J Sports Med. 1988 Mar-Apr;16(2):123-9.

[39] Ko TS, Joung HB. Effects of static stretching and Evjenth-Hamberg stretching on isokinetic strength in the knee. J Korean Soc Physical Ther. 2006;18(5):43-51.

[40] Kim GH. Comparison of duration of flexibility of hamstring after static, dynamic, proprioceptive neuromuscular facilitation stretching. Doctor's Degree. Hanyang University. 2002.

[41] Björklund M, Hamberg J, Crenshaw AG. Sensory adaptation after a 2-week stretching regimen of the rectus femoris muscle. Arch Phys Med Rehabil. 2001;82(9): 1245-50. 